\title{
Morphin statt schwache Opioide bei moderaten Krebsschmerzen
}

Hintergrund und Fragestellung: Die WHO-Leitlinien für die Behandlung von Krebsschmerzen empfehlen drei sequenzielle Stufen einer analgetischen Treppe. Es gibt jedoch keine ausreichenden Daten dazu, ob moderater Schmerz mit schwachen Opioiden der Stufe II oder mit niedrig dosierten starken Opioiden der Stufe III behandelt werden sollte.

Patienten und Methodik: In einer multizentrischen, 28 Tage währenden, offenen, randomisierten, kontrollierten Studie wurden Patienten mit moderaten Krebsschmerzen entweder mit schwachen Opioiden (Stufe II) oder niedrig dosiertem Morphin (Stufe III) in retardierter Form behandelt. Primärer Endpunkt war die Zahl der Patienten mit einem Ansprechen, definiert als eine 20\%ige Reduktion der Schmerzintensität auf einer numerischen Ratingskala.

\section{Originalie}

Bandieri E et al. Randomized Trial of Low-Dose Morphine Versus Weak Opioids in Moderate Cancer Pain. J Clin Oncol. 2016;34(5):436-42.
Ergebnisse: Insgesamt wurden 240 Krebskranke in die Studie aufgenommen, 118 erhielten niedrig dosiertes Morphin, 122 ein schwaches Opioid der Stufe II. Ein Ansprechen wurde bei $88,2 \%$ der Patienten unter niedrig dosiertem Morphin und bei
57,7\% unter schwachen Opioiden erreicht (Odds Ratio 6,18, $95 \%$-Konfidenzintervall 3,12-12,24; $\mathrm{p}<0,001)$. Bereits nach einer Woche hatten mehr Patienten in der Gruppe mit niedrig dosierter Morphin-Gabe auf die Schmerztherapie angesprochen (80,9 vs. $43,6 \% ; \mathrm{p}=0,001)$. Darüber hinaus erzielten über die gesamte Beobachtungszeit mehr Patienten in der Morphin-Gruppe ein Ansprechen. Zudem wurden eine relevante $(\geq 30 \%)$ und wesentliche Schmerzreduktion ( $\geq 50 \%$ ) signifikant häufiger bei Patienten unter niedrig dosiertem Morphin erreicht (82,7 vs. 47,0\% bzw. 75,5 vs. $41,9 \%$; $p$ jeweils $<0,001$ ). Außerdem war die allgemeine Verfassung der Patienten - bestimmt mithilfe des „Edmonton Symptom Assessment Systems“, einer umfassenden Symptom-Bewertung - in der Morphin-Gruppe besser. Wegen einer unzureichenden Analgesie musste die Therapie bei Patienten in der Guppe mit schwachen Opioiden häufiger angepasst werden. Unerwünschte Arzneimittelwirkungen waren in beiden Gruppen vergleichbar.

Schlussfolgerungen der Autoren: Bei Krebskranken mit moderaten Schmerzen konnte die Schmerzintensität durch eine Therapie mit niedrig dosiertem Morphin signifikant besser und früher gemindert werden als durch schwache Opioide; und dies bei vergleichbarer Verträglichkeit der Therapie.

Schlüsselwörter: Chronische Tumorschmerzen - schwache Opioide - Morphin - WHO-Stufenleiter

\section{- Kommentar von Ulrich R. Kleeberg, Hamburg}

\section{"Bei Tumorschmerzen ist das Zweistufenkonzept sinnvoll"}

Die dreistufige Therapieleiter der Weltgesundheitsorganisation (WHO) sieht vor, dass die Wahl der Analgetika-Therapie je nach Intensität der Krebsschmerzen von der niedrigsten Stufe I (Nicht-Opioide) über die Stufe II (schwache Opioide wie Kodein, Tramadol) dann zur Stufe III (starke Opioide wie Morphin, Fentanyl, Oxycodon) angehoben wird. Stattdessen wird inzwischen von der European Association for Palliative Care (EORTC) ein Zweistufen-Konzept (WHO-Stufe I und WHO-Stufe III) ohne die schwachen Opiode der Stufe II empfohlen [2]. Diese multizentrische Arbeit von Elena Bandieri und Kollegen bestätigt nun einmal mehr, dass das Festhalten an der dreistufigen Therapieleiter der WHO in der Onkologie nicht angemessen ist.

Entscheidend für den Erfolg der Behandlung von Krebsschmerzen bleibt grundsätzlich neben einer vorsichtig einschleichenden Dosierung eine angemessene, vorbeugende Behandlung der unerwünschten Arzneimittelwirkungen. Bedacht werden müssen stets auch eine kausal gegen den Tumor gerichtete palliative Intervention und die Kombination mit adjuvanten Medikamenten und Maßnahmen. Dass die Stufe Il der WHO-Stufenleiter übersprungen werden kann, ist da- bei eine wichtige Erkenntnis für eine optimale Behandlung chronischer Krebsschmerzen. Dieses Vorgehen ist notgedrungen mit einem etwas höheren bürokratischen Aufwand verbunden. In unserer täglichen Routine ist dieser aber problemlos zu bewältigen und unbedingt zu empfehlen.
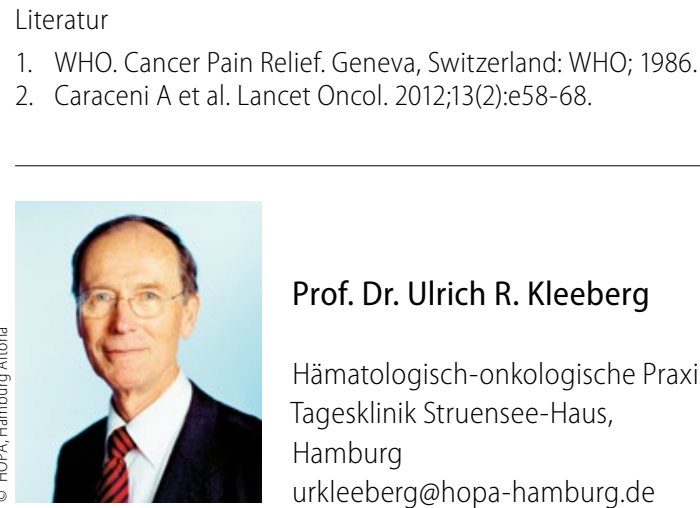

Prof. Dr. Ulrich R. Kleeberg

Hämatologisch-onkologische Praxis Altona, Tagesklinik Struensee-Haus, Hamburg urkleeberg@hopa-hamburg.de 